\title{
Fundación, esplendor y COlapso de la iglesia de SAN Miguel de Piura (1534-1578), Primer TeMplo del Perú
}

\author{
César W. Astuhuamán Gonzáles ${ }^{a}$
}

\begin{abstract}
Resumen
Esta investigación analiza la infraestructura e instituciones imperiales desarrolladas por los incas y españoles para controlar y administrar la región Piura mediante Piura La Vieja (o San Miguel de Piura). Dos preguntas son abordadas: ¿Cuáles fueron las funciones de las edificaciones institucionales? ¿ ¿cómo sus funciones fueron cambiando desde los periodos prehispánico al Colonial Transicional? Para responderlas, combiné fuentes documentales y evidencias arqueológicas. Los españoles fundaron San Miguel en 1534 y, en 1539, una iglesia católica fue construida según fuentes documentales, la cual operó hasta 1578. Durante el trabajo de campo de 2005, 2008 y 2011, se excavó una edificación, construida sobre una prehispánica, localizada al sur de la plaza central, y se registraron componentes arquitectónicos de una iglesia: atrio, puerta principal, campanario o espadaña, muro perimétrico, altar y sacristía. La importancia de la iglesia radica en que es la unica edificación sobreviviente de las siete iglesias tempranas construidas en el Perú antes de 1540. Este artículo es organizado en cinco partes: la primera está dedicada a explorar el periodo prehispánico (1800 a.C.-1532 d.C.); la segunda, al establecimiento hispano de San Miguel de Piura; la tercera y cuarta, al ascenso y colapso del sitio; finalmente, se presentan algunas conclusiones preliminares y futuras investigaciones son presentadas.
\end{abstract}

Palabras clave: Periodo Colonial Transicional, Piura, edificación religiosa, culto, arquitectura

\section{Abstract \\ FOUNDATION, SPLENDOUR, AND COLLAPSE OF THE SAN MIGUEL DE PIURA CHURCH (1534- 1578), THE FIRST CATHOLIC CHURCH OF PERU}

The aim of this research is to analyze the imperial infrastructure and institutions developed by the Inca and Spaniards to control and administer the Piura Region through the major center of Piura La Vieja (or San Miguel de Piura). This study leads with two main questions: What were the functions of the institutional buildings at Piura La Vieja, and how did these functions change from the pre-Hispanic to Transitional Colonial periods? To answer these questions, I developed a methodology to concede the limited documentary sources and surviving archaeological evidence. The Spaniards established San Miguel de Piura in 1534 and according to early documentary sources, a Catholic church was built circa 1539, which operated until 1578. During the 2005, 2008 and 2011, field seasons at Piura La Vieja we identified and excavated a structure to the south of the central plaza, oriented from North-West to South-East. The structure was identified and recorded as the architectural remains of a church, including an atrium, main entrance, tower, outer wall, altar, and sacristy. This church was built over a pre-existing pre-Hispanic building which followed a different axial orientation. The church of San Miguel de Piura is significant in that it is the only surviving building of the first seven churches built in Peru before 1540. This article is organized in five parts: the first is dedicated to explore the pre-Hispanic Period (1800 a.C.-1532 d.C.), the second to the Spanish establishment of San Miguel de Piura, the third to the brief rise of San Miguel, the fourth to the collapse of the site. Finally, some preliminary conclusions and directions for further research are presented.

Keywords: Transitional Colonial Period, Piura, religious building, cult, architecture

${ }^{a}$ Departamento de Arqueología, Universidad Nacional Mayor de San Marcos

Correo electrónico: astuhuaman@hotmail.com 


\section{Introducción}

Los templos han sido considerados los centros del mundo, espacios donde los hombres se encuentran con la deidad, a la que han dedicado sus mejores esfuerzos constructivos, donde los cultos y rituales religiosos pueden ser investigados a partir de la cultura material (Eliade 1998; Renfrew y Bahn 1998; Rappaport 2001). Las iglesias cristianas fueron entendidas como los centros de las ciudades, estas, a su vez, concebidas como una representación del universo. En el siglo XV, cuando las sociedades europeas iniciaron la colonización del Nuevo Mundo, llevaron consigo la cruz y la espada, y fundaron ciudades e iglesias en otras tierras que tenían ya sus propios templos y deidades.

A diferencia de El Caribe, con las primeras ocupaciones europeas en La Isabela entre 1493 y 1498 (Deagan y Cruxent 2002), y Santo Domingo en 1502 (Deagan 1996), las investigaciones de arqueología histórica acerca de los primeros asentamientos europeos y sus edificaciones religiosas han sido escasas en América del Sur.

En este artículo, se presentan los resultados de las temporadas de investigación 2008 y 2011, orientados al entendimiento de la fundación, esplendor y colapso de la iglesia de San Miguel de Piura (localizada en Piura La Vieja) en el contexto del proceso de evangelización y transformación del pensamiento andino en el extremo Norte del Perú. Uno de los objetivos de esta investigación fue contrastar las hipótesis acerca de la localización y caracterización arqueológica de algunas edificaciones documentadas históricamente en Piura La Vieja, en especial, de la iglesia.

Piura La Vieja es parte de la región Piura, y su localización espacial en el valle alto del río Piura, entre quebradas y cruces de caminos, entre la costa y la sierra, es clave para entender su rol en la historia regional (Fig. 1). El área de investigación se ubica políticamente en el centro poblado Piura La Vieja, distrito de La Matanza, provincia de Morropón, región Piura, y a una altitud que varía de 90 a 145 metros sobre el nivel del mar, en la margen derecha del río Piura, al pie de la cordillera. Piura La Vieja tiene una larga secuencia de ocupación, que se remonta desde el Período Inicial hasta la actualidad (Hocquenghem 1998; Rodríguez 2006). El área era recorrida por la red de caminos inca, a lo largo de la cual se encontraban los centros locales que luego fueron ocupados por los europeos; en uno de estos centros, se fundó San Miguel en 1534.

\section{Piura La Vieja durante tiempos prehispánicos}

Las investigaciones arqueológicas en Piura La Vieja se iniciaron en 1999, producto de un convenio de la Universidad Politécnica de Madrid con la Universidad de Piura, y han continuado hasta el año 2012. Los reconocimientos de superficie han permitido elaborar un plano de Piura La Vieja y realizar excavaciones en sus diversos sectores, las que han permitido comprender la secuencia de ocupación del sitio a partir de rasgos formales y análisis estratigráfico (Fig. 2).

La ocupación preinca de Piura la Vieja había sido reportada anteriormente a partir del material cerámico registrado en superficie (Hocquenghem 1998; Montenegro 2005) y durante las excavaciones de la temporada 2005-2006 (Rodríguez 2006). Los resultados de las excavaciones desarrolladas durante la temporada 2011 han confirmado la ocupación preinca del sitio, en particular, en el Montículo Prehispánico 1 (Astuhuamán 2012). Así mismo, la presencia de cerámica de los estilos piura, chimú y sicán en las excavaciones en el Sector ES-1 nos muestra la larga secuencia de ocupación de Piura La Vieja, ya planteada previamente. Al menos, tres momentos de ocupación han sido identificados en el Montículo Prehispánico 1: antes, durante y después de la ocupación europea. La primera ocupación ha sido evidenciada por fragmentos de cerámica de los estilos piura y chimú-local, recuperados durante las excavaciones. Piura La Vieja fue el principal centro local en la jerarquía de asentamientos de la cuenca alta del río Piura durante el Período Intermedio Tardío (c. 1000-1400 d.C.), según Montenegro (2010).

Con respecto a la arquitectura monumental prehispánica, en torno a la plaza central, se presenta una alta concentración de plataformas masivas, algunas de ellas escalonadas y con rampas que 


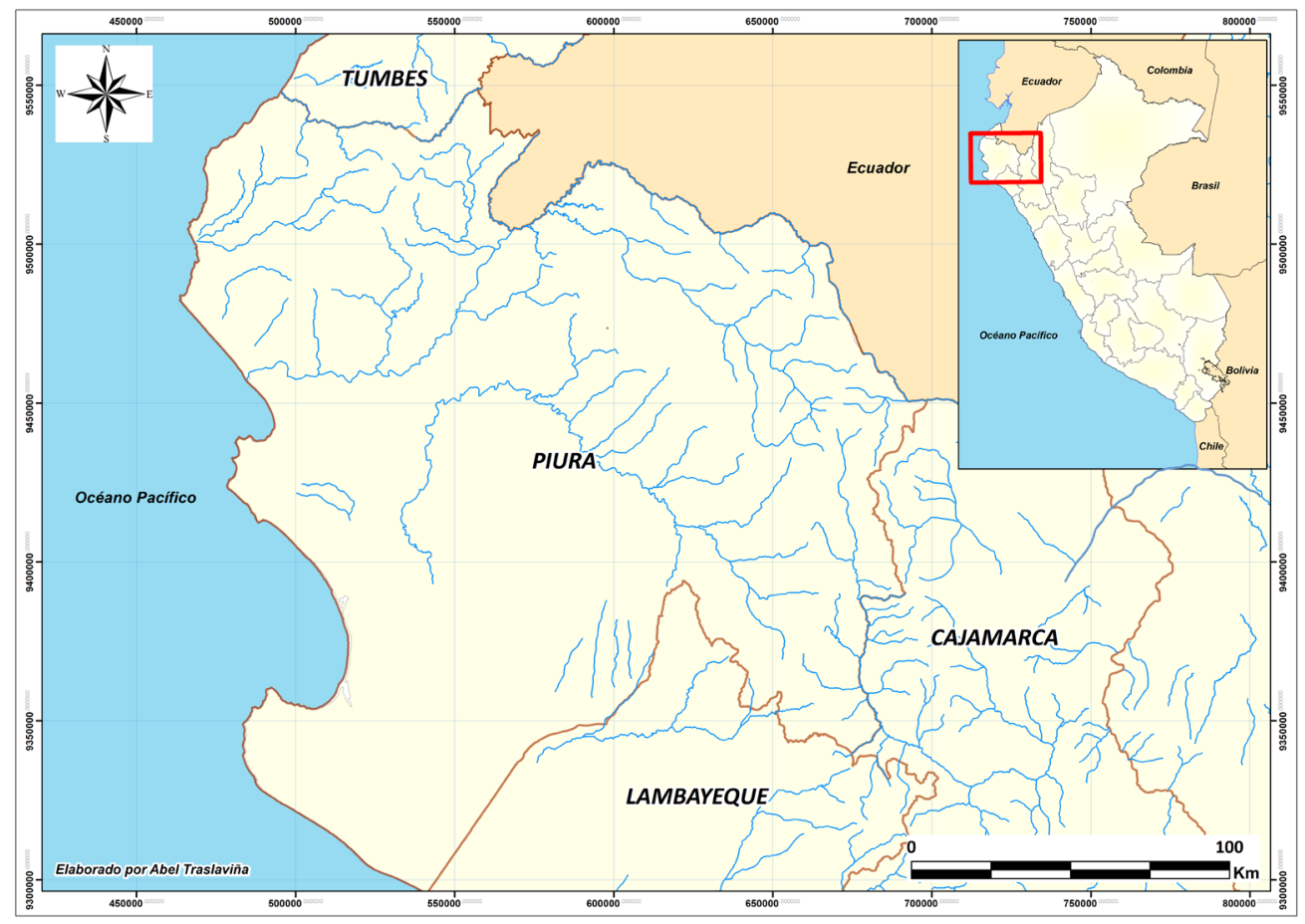

Figura 1. El extremo norte de Perú y el sur de Ecuador (Mapa por C. Astuhuamán).

corresponden a las tradiciones arquitectónicas norteñas. Una de ellas es el Montículo Prehispánico 1 , cuya construcción aterrazada fue posible delinear a partir de las excavaciones y limpieza de perfiles en dicho sector.

La ocupación inca de Piura La Vieja había sido reportada anteriormente, principalmente, a partir del material cerámico registrado en superficie (Hocquenghem 1998; Montenegro 2005, 2010; Rodríguez 2006; Astuhuamán 2008). Los resultados de las excavaciones desarrolladas durante la temporada 2011 han corroborado nuevamente la ocupación inca del sitio, en particular, la presencia de material cerámico inca en los sectores ES-1 y MP-1. Una mirada a Piura La Vieja más allá de los sectores excavados, ES-1 y MP-1, proporcionará algunas hipótesis acerca de la presencia inca en el sitio.

Uno de los objetivos de esta investigación era proseguir la evaluación del potencial arqueológico de los principales sectores de Piura La Vieja. Esto implicó analizar las edificaciones e instituciones asociadas para controlar y administrar la región desde tiempos preinca hasta el Período Colonial Transicional (1534-1578). Así, se evaluó cómo ambas estuvieron relacionadas con la forma y la función de la organización provincial, y con la definición del rol de la infraestructura y las instituciones dentro de la organización de los sucesivos Estados que gobernaron la región Piura. Si bien nuestra investigación se concentró en el Período Colonial Transicional, también, se obtuvo información acerca de tiempos prehispánicos, lo que ha permitido entender mejor la ocupación europea de Piura La Vieja (Fig. 3).

Utilizando información etnohistórica y asumiendo que los sitios incas más extensos fueron los centros de las jurisdicciones inca, denominados provincias por los cronistas españoles, sugiero que cada uno de ellos representaría una provincia diferente. Esta hipótesis se basa en un marco teórico (cf. Renfrew 1975; Renfrew y Level 1979) que es ampliamente seguido por quienes han investigado la organización provincial inca (por ejemplo, D’Altroy 1992; Matos 1994; Morris 1998; Cornejo 2000; Williams 2002-2005). Así, Piura La Vieja fue el centro de una jurisdicción inca, 


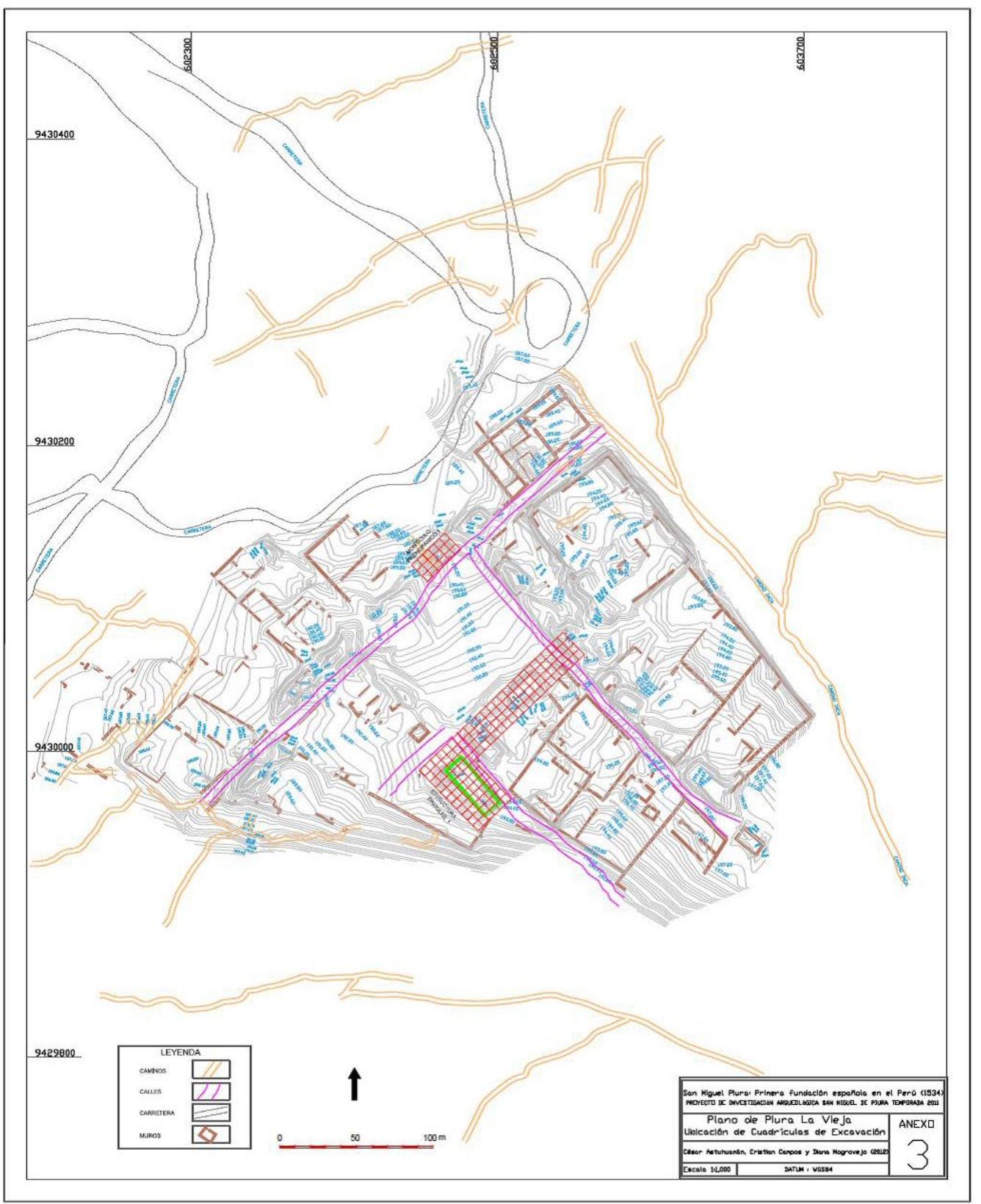

Figura 2. Plano de Piura La Vieja y distribución de cuadriculas de excavación (Fuente: Astuhuamán 2012).

al igual que Caxas, Aypate, Huancabamba y Mitupampa lo fueron en la sierra de Piura. Piura La Vieja y Pohechos eran los centros provinciales inca más importantes en la costa de la región Piura. La primera fue asociada al camino longitudinal de la costa y hacia el cual se dirigía un camino transversal proveniente de Caxas (Astuhuamán et al. 2003; Astuhuamán 2008). Ello permitió situar a Piura La Vieja en el contexto de la red de centros y caminos incas que permitieron administrar la región Piura durante el tiempo del Tawantinsuyu.

Se ha registrado que la planta general de Piura La Vieja corresponde a un patrón ortogonal y que los edificios más importantes del asentamiento español, los más cercanos a la plaza central, 


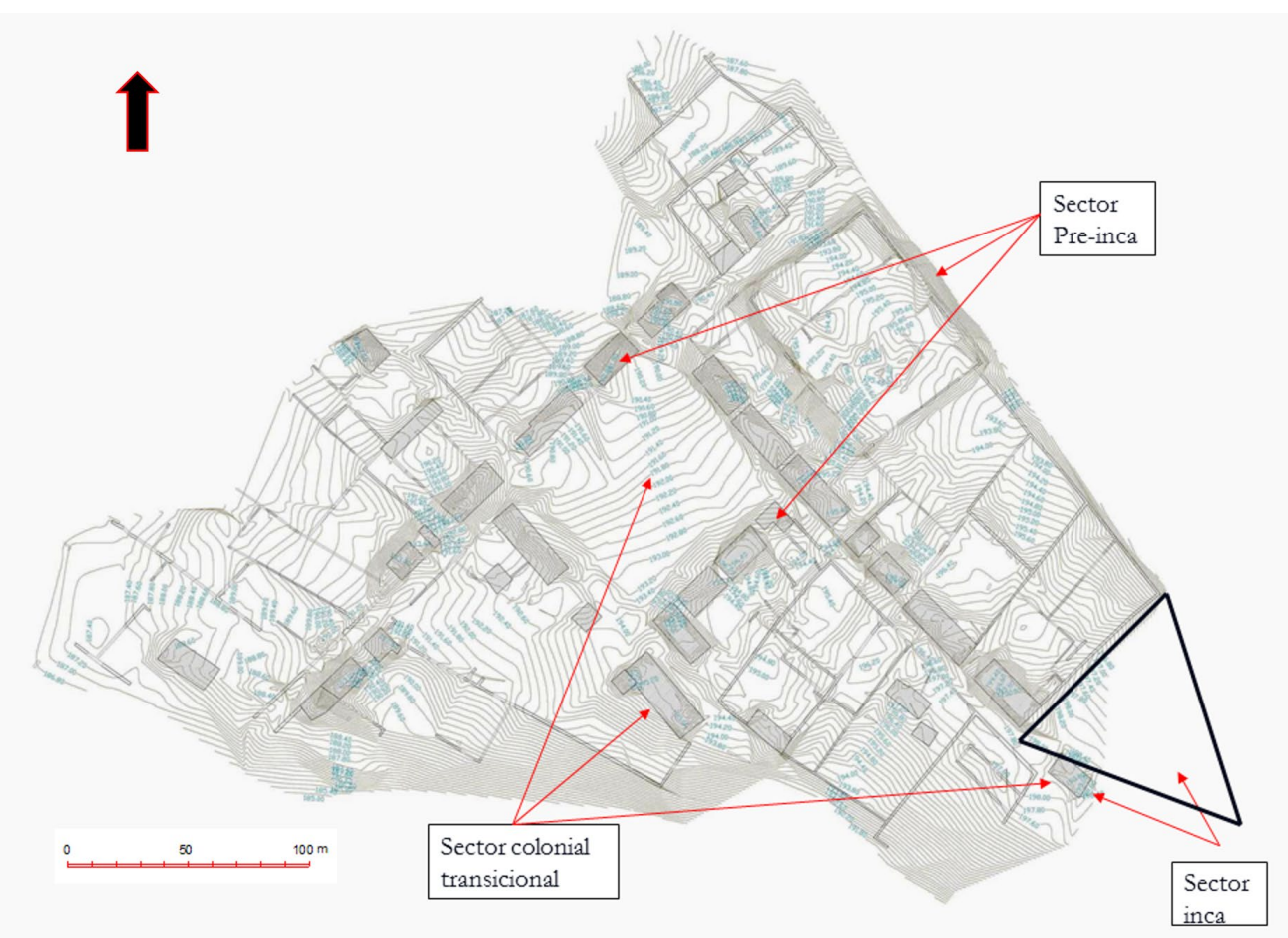

Figura 3. La secuencia de ocupación humana en Piura

La Vieja (Plano por C. Astuhuamán).

habrían sido edificados sobre plataformas masivas (Rodríguez 2006: 67; Montenegro 2010). Estas plataformas están integradas al plan ortogonal del sitio. Aunque no son parte de la tradición constructiva europea, tampoco forman parte de la arquitectura inca, sino que son propias de las tradiciones arquitectónicas prehispánicas norteñas (v.g. Sicán y Piura). Piura La Vieja presenta la más alta concentración de plataformas del valle del río Piura, lo cual sería un indicador importante para ser considerado el centro tallán de la región Piura (Montenegro 2010) y explicaría por qué los incas construyeron allí su centro provincial luego de 1400 d.C.

Lo que se encuentra en discusión es la antigüedad del patrón ortogonal de la traza urbana de Piura La Vieja ( $c f$. Rodríguez 2006), dado que el patrón ortogonal y el damerado fueron usados tanto por los incas como por los españoles (Hyslop 1990; Van Buren et al. 1993: 139-140). Dos patrones definieron la traza urbana de los centros incas: el patrón ortogonal y el patrón radial alrededor de un centro (Hyslop 1990: 191). Una característica común en los centros incas con patrón ortogonal es que el sector de edificaciones mejor elaboradas, de mayor tamaño, y diferente al resto de canchas que integraban el sitio inca, se encuentra en un extremo del asentamiento y no en el centro geométrico del mismo (ibid:: 201). Sin embargo, en Piura La Vieja, los edificios con mayor volumetría se encuentran en el centro geométrico del sitio y fue allí donde posteriormente se construyó el centro español de San Miguel de Piura (Fig. 4).

Algunos ejemplos del uso sucesivo del patrón ortogonal inca y del damerado español de las reducciones, y de la dificultad para definir en qué período fueron fundados son los sitios de Nieve Nieve, Torata Alta y Chucuito. En estos centros incas con patrón ortogonal, se instalaron las reducciones de indios siguiendo la traza del damero hispano, que coincide con el patrón ortogonal inca. Este no sería el caso de Piura La Vieja, pues su reconstrucción europea es precedente al período toledano.

A pesar de las similitudes del patrón ortogonal utilizado por los incas y españoles, Hyslop ha planteado algunas características que los podrían diferenciar. En primer lugar, la traza inca es 


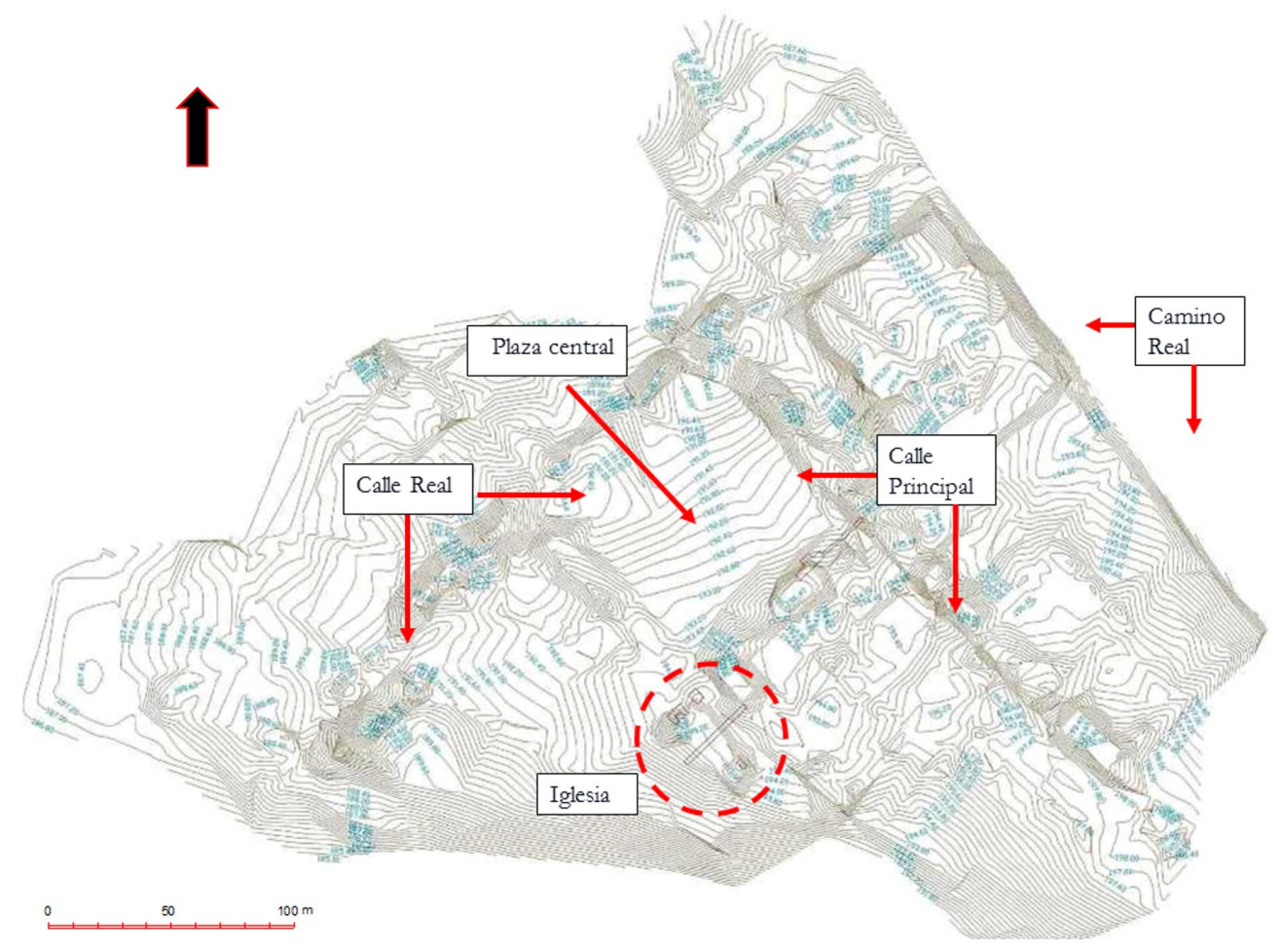

Figura 4. Plano de distribución de las calles y edificaciones de San Miguel de Piura (Plano por C. Astuhuamán).

irregular e interrumpida por accidentes geográficos, mientras la traza europea es conformada por calles paralelas, las manzanas son de igual tamaño y la traza no es afectada por el relieve terrestre. En segundo lugar, el patrón ortogonal es utilizado en la totalidad del asentamiento español; en cambio, la traza ortogonal inca es combinada con otros principios de organización (como el patrón radial). En tercer lugar, las plazas incas se localizan en los extremos del asentamiento, mientras que las plazas hispanas se encuentran en el centro (Hyslop 1990: 192, 201; Van Buren et al. 1993: 139-140). Sin embargo, no todas las reducciones de indios presentan un patrón ortogonal regular, y sus iglesias podían situarse también en el extremo del asentamiento ( $v$.g. Nieve Nieve) o no estar cerca a la plaza (Van Buren et al. 1993: 140). Así, distinguir entre el patrón ortogonal inca y español a partir solo de los planos arquitectónicos es difícil. Esto, tal vez, podría ser aclarado mediante excavaciones sistemáticas, como en el caso de Piura La Vieja, pero teniendo en cuenta que Piura La Vieja no fue organizada como una reducción de indios, sino que era una ciudad de encomenderos.

La información registrada durante la temporada 2011, así como la registrada durante las temporadas 2005 y 2008 (Rodríguez 2006; Astuhuamán 2009), el análisis de las fotografías aéreas y las ortofotografías de satélite nos sugieren que el trazado semirregular de Piura La Vieja podría obedecer al patrón ortogonal inca y está definido por la existencia de al menos dos vías de comunicación interna prehispánicas, sobre las que la traza urbana inca se organizó siguiendo un eje de orientación aproximada noroeste y sureste, y que luego fueron reutilizadas por los espańoles como calles. Al estar el patrón ortogonal inca en Piura La Vieja emplazado sobre una terraza aluvial elevada de forma triangular alargada, esto nos condujo a plantear la localización del sector inca más importante de Piura La Vieja en un extremo del asentamiento y no en el centro del mismo, siguiendo otros casos documentados por Hyslop (1990). Así, el principal sector inca de Piura La Vieja se encontraría en la parte triangular alargada del sitio, en torno a una supuesta plaza triangular. Esta forma de plazas también ha sido reportada en otros centros inca (cf. Hyslop 1990). 
Hacia esta plaza, convergería una de las dos principales vías de comunicación interna del sitio, y, desde esta plaza, saldría un pequeño camino que descendía hacia el camino inca que recorre lateralmente el sitio con rumbo a Serrán y Caxas. La hipótesis de la plaza triangular requiere de un reconocimiento sistemático de esta parte de Piura La Vieja y de un levantamiento topográfico de la misma con la finalidad de ubicar los muros de contención que la habrían delimitado.

Un reconocimiento preliminar en torno a la hipotética plaza triangular permitió identificar las siguientes edificaciones: una plataforma escalonada con rampa y un recinto alargado que fue reocupado por los españoles. El primero podría ser un templo; y el segundo, una kallanka inca. Un levantamiento planimétrico y un registro sistemático de las edificaciones en torno a la hipotética plaza triangular, así como excavaciones, podrían aclarar la naturaleza y secuencia de ocupación de dichas edificaciones. La localización de dichos edificios, alejados de la concentración de plataformas masivas, sugiere que el sector inca de edificios que rodean la plaza triangular habría desplazado al sector principal del centro local preinca (situado en torno a la concentración de plataformas masivas alrededor de la plaza cuadrangular); de ese modo, habría quedado lejos del centro geométrico del sitio. Durante su dominio del Alto Piura, los incas movieron el centro del poder en Piura La Vieja.

\section{La convulsionada fundación de San Miguel de Piura}

Los primeros intentos de colonización y evangelización hispana en el Tawantinsuyu empezaron en 1528, durante el segundo viaje de Pizarro. Pedro de Candia, armado con una espada, arcabuz y una cruz de madera, desembarcó en el centro inca de Tumbes (Cieza 1997b [1554]: 63; Oliva 1998 [1630]: 114). El impresionante relato que Candia hiciese de Tumbez condujo a que, en la Capitulación de Toledo (1529), se propusiese a Hernando de Luque como obispo de Tumbez (Cieza 1997b [1554]: 84) y lo nombrasen protector de indios. Sin embargo, al retornar Pizarro en 1532, Tumbez estaba ya destruido por las guerras internas, por lo que se fundó el primer centro hispano en Tangarará, valle bajo del Chira, donde se celebró la primera misa. Al respecto, cabe anotar que aquel San Miguel tuvo una corta vida (Elías 2008: 13).

Luego de estos dos intentos frustrados de establecimiento español, San Miguel fue trasladada en 1534 por Diego de Almagro hacia Monte de los Padres, en el valle medio del río Piura, cerca al pueblo del curaca local (Vela 2000; Villanueva 2001; Vela y Villanueva 2002; Villanueva et al. 2002: 289; Elías 2008: 13). Según los documentos históricos, la iglesia de San Miguel se construyó entre 1535 y 1539 por fray Vicente Valverde por encargo de los reyes de España. Así, en su carta al emperador, fechada el 20 de marzo de 1539, señala que eran siete las iglesias levantadas (Cuzco, Los Reyes, Trujillo, San Miguel, Portoviejo, Santiago de Guayaquil y en San Juan de la Frontera de Huamanga). Sobre ellas, informa, "[...] he distribuido todos los ornamentos que de allí truxe, proveyendo a cada una, según su necesidad [...]» (Valverde 1539, citado en Vargas 1953: 120). Vargas nos proporciona una idea de las sencillas iglesias de este tiempo, teniendo como referencia la de Huamanga: «[...] Toda ella se reduce a un rectángulo de piedra y adobe, sin adornos ni ventanas y cubierta con un rústico techo de troncos y cañas gruesas, entretejidas. A lo largo de uno de los muros corre un poyo de mampostería que debió servir de asiento a los fieles, pero a la entrada no deja de atraer la atención el amplio arco de piedra que da paso al templo y a cuyo flanco se levanta la única torre, rústica y sencilla como todo el conjunto» (Vargas 1953: 120).

En 1540, un sacerdote de Sevilla (Juan Funes) fue nombrado por el rey para estar a cargo de la iglesia de San Miguel (Patronato 278, N2, R142) y, hacia 1544, un grupo de 40 niños estaban al servicio de la iglesia de San Miguel para catequizar a otros nińos de la zona (Estenssoro 2003: 42).

Fue durante la convulsionada época comprendida entre 1533 y 1548 que San Miguel, hoy Piura La Vieja, empezó su breve consolidación como el centro de avanzada española para la conquista del Tawantinsuyu. Dicho afianzamiento se inició con la construcción de los edificios institucionales en la nueva ciudad: iglesia matriz, Convento de Mercedarios, casas del Cabildo, hospital y otros. De estos edificios, las excavaciones desarrolladas durante las temporadas de investigación 2005, 2008 y 2011 han permitido contrastar la hipótesis acerca de la identificación de la 


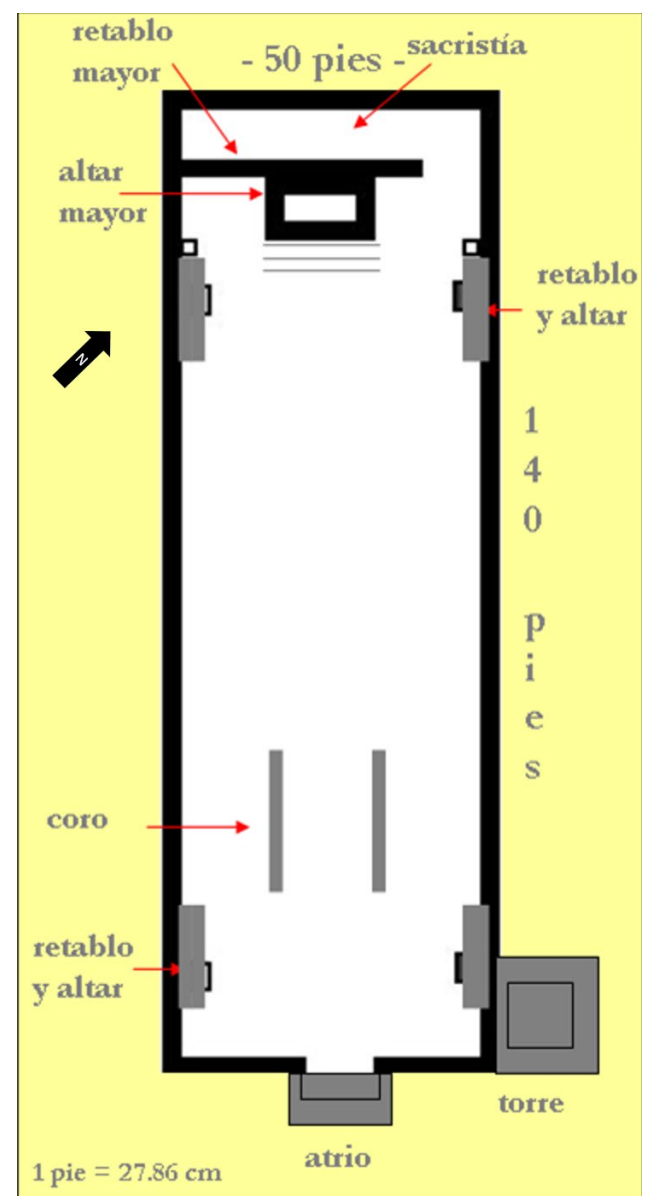

Figura 5. Evidencias que indican que el Sector ES-1 era la iglesia de San Miguel de Piura (Plano por C. Astuhuamán).

iglesia matriz. De San Miguel, Cieza de León escribió que aquí "[...] se hizo el primer templo a honra de Dios nuestro señor [...]» (1973 [1551]: 155).

Las excavaciones en el Sector Estructura Singular 1 (ES-1) y el hallazgo de las siguientes evidencias indican que se trataba de una iglesia con las siguientes características: un muro perimetral de planta rectangular; un altar escalonado; entierros humanos extendidos al interior de la edificación, uno de ellos orientado hacia el altar; retablos laterales situados alternadamente en los costados internos de la edificación; un espacio situado a la espalda del altar mayor que habría sido utilizado como sacristía; un montículo elevado situado en un extremo del frontis, construido con técnica prehispánica, el cual fue usado como la base de una espadaña o un campanario; la proporción modular (de una a tres) en las dimensiones de ancho (50 pies) y largo (140 pies) de la edificación, típica de edificaciones religiosas europeas; su ubicación en una esquina de la plaza; el atrio; y una entrada principal (Fig. 5).

Respecto a estas evidencias, el altar escalonado presenta siete $\mathrm{u}$ ocho peldaños pintados de color blanco que conducen desde la nave de iglesia hacia la plataforma superior que conformaba la mesa del altar, la cual ha sido mayoritariamente saqueada. En la parte posterior de la plataforma, se observan los restos del muro que conformaba el retablo mayor (Fig. 6). El altar se halla más próximo al muro norte de la iglesia, no en el eje longitudinal de la iglesia; de este modo, genera un espacio entre el altar y el muro sur de la iglesia, el cual conforma un pasadizo que conduce desde la nave hacia la sacristía (Fig. 7). 


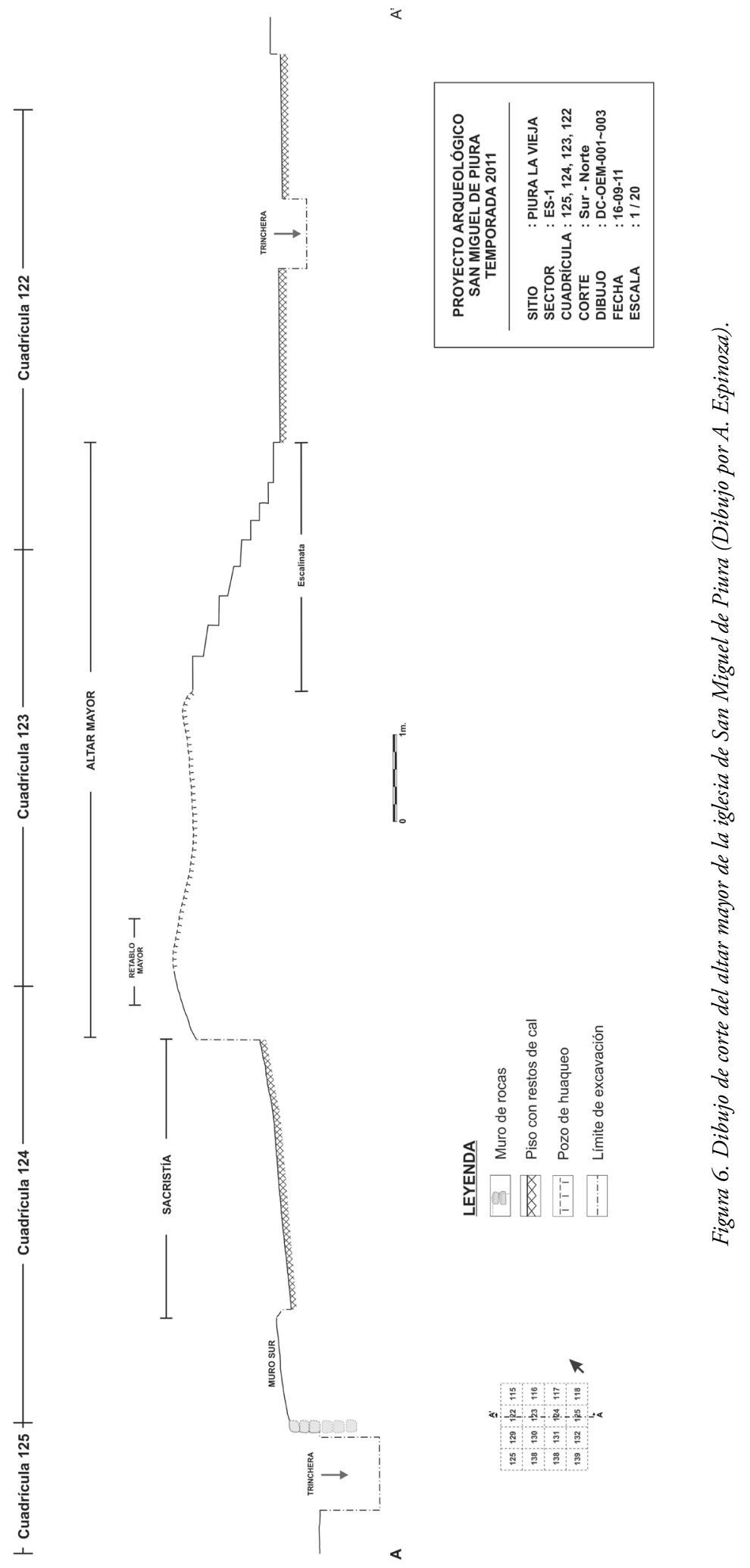




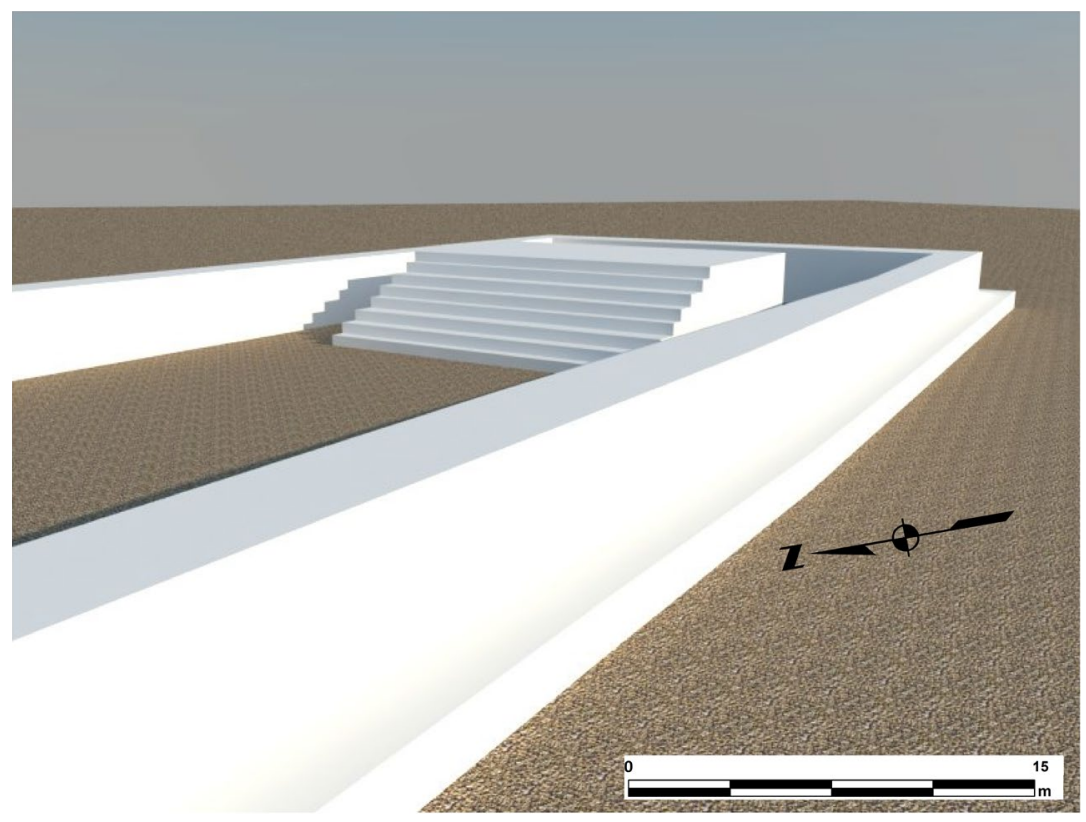

Figura 7. Reconstrucción hipotética del altar mayor de la iglesia de San Miguel d Piura, observar el pasadizo hacia la derecha que conduce a la sacristía (Dibujo por D. Mogrovejo).

Sin embargo, existen algunos detalles atípicos que destacan en la iglesia (Estructura Singular 1) y en las otras edificaciones situadas alrededor de la plaza, que cuestionan su carácter totalmente español. El primero es su orientación general, noroeste y sureste, la cual no coincide con la de los ejes cardinales frecuentemente registrada en iglesias tempranas y en las tumbas cristianas registradas al interior ( $c f$. Schülke 1999). El segundo es la ubicación de la iglesia en torno a la plaza. No se encuentra en uno de los cuatro lados de esta, sino en una esquina de la plaza y al costado de una estructura elevada conformada por plataformas escalonadas de origen prehispánico (que sí se sitúa en uno de los cuatro lados de la plaza). El tercero es la construcción parcial de la iglesia sobre muros o plataformas de origen prehispánico, registrados durante las temporadas 2005, 2008 y 2011. El cuarto es la presencia de material arqueológico prehispánico mezclado con cerámica transicional y artefactos europeos. El quinto es una estructura arquitectónica al interior de la nave.

Con respecto al primer detalle atípico, esta característica deriva de la orientación del patrón ortogonal inca del asentamiento e indica que la traza europea de San Miguel (y de la iglesia en particular) se vio afectada por la traza urbana inca preexistente.

En cuanto al segundo detalle, debido a que el patrón ortogonal inca no presenta una plaza central, entonces, es válido preguntarse cuándo se construyó la plaza central que actualmente se reconoce como parte del damero español. Si la plaza se construyó durante la fundación de San Miguel (probablemente, a partir de la demolición y allanamiento de sectores prehispánicos) y desde allí se ordenaron los solares que la integran, entonces, la iglesia podría haberse construido en uno de los lados de la plaza y sobre el montículo prehispánico, al costado del cual se encuentra. Sin embargo, si la iglesia se construyó antes de la construcción de la plaza, entonces, su ubicación en relación con esta no habría estado clara inicialmente y pudo ser excéntrica (tal como resultó siendo al construirse en una esquina).

La ubicación de la iglesia al costado de una estructura elevada y escalonada de origen prehispánico, que pudo ser construida sobre esta para obtener mayor altura y preeminencia, sugiere que su localización pudo ser negociada con los líderes del grupo local, pues, durante la gradual desintegración del Estado inca, los líderes locales (tallanes) volvieron a adquirir poder, y se convirtieron 


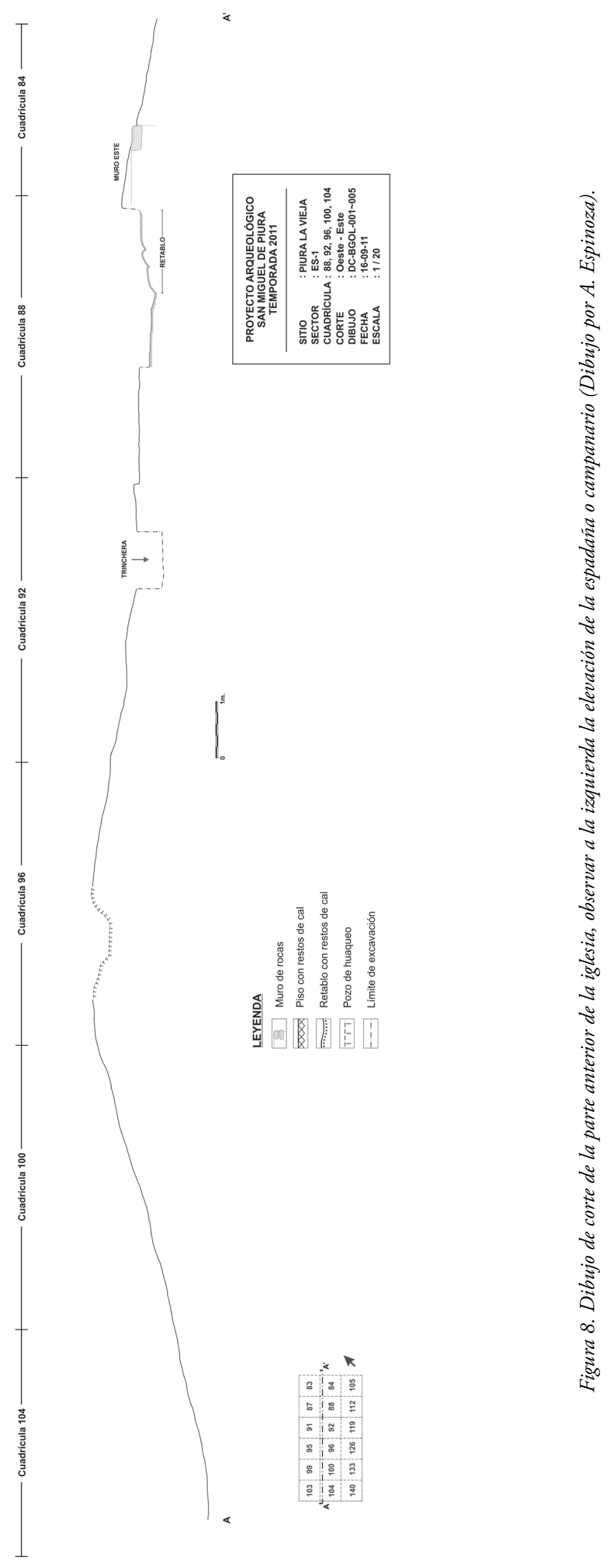


en aliados de los españoles y enemigos de los incas, en especial, de la facción de Atahualpa. Esto explicaría por qué la iglesia no se construyó sobre el montículo prehispánico local: este sería un lugar sagrado para los aliados locales. Esta situación se puede entender mejor si se considera que, en el convulsionado período que va de 1532 a 1548, lo más importante para los espańoles era tener como aliados a los señores locales; era una etapa de tolerante convivencia religiosa (Duviols 1977: 109-114) o de antagonismo tolerante.

Las dispersas aldeas de la región Piura permanecieron bajo la autoridad de los curacas aún durante los tiempos del Pacificador La Gasca (1547-1550). Por su ubicación marginal, el proceso de evangelización pudo verse poco afectado por las constituciones del Primer Concilio de Lima de 1551 (Duviols 1977: 97-100; Huertas 1996; Estenssoro 2003: 64-71). Será mucho después, durante el gobierno del virrey Francisco de Toledo (1569-1581), que fueron implementadas nuevas regulaciones para instalar a las poblaciones locales en reducciones (con iglesia incluida) y, recién en el siglo XVII, iniciaron sistemáticamente las campañas de extirpación de idolatrías (Duviols 1977), cuando la ciudad de San Miguel de Piura ya estaba abandonada y se había convertido en Piura La Vieja.

El tercer detalle atípico es la construcción parcial de la iglesia sobre muros o plataformas de origen prehispánico. No es clara la antigüedad de estas evidencias, pero sí es claro que se prefirió afectarlas antes que a la estructura tronco piramidal que se encuentra al costado de la iglesia. Además, se observa que el frontis de la antigua edificación tenía mejor acabado que el de la iglesia, evidencia de la precaria presencia del Estado español en San Miguel de Piura. Así, el hallazgo de muros debajo de la iglesia indica que existía una edificación prehispánica y que esta fue allanada para construir la iglesia encima, y que se aprovechó una esquina de la antigua plataforma para construir allí la espadaña o el campanario, y así obtener la altura necesaria para colocar la campana (Fig. 8).

Con respecto al cuarto detalle atípico, el material arqueológico del relleno (v.g. cerámica, metales) registrado tanto en el piso de la iglesia como en los apisonados del montículo situado en una esquina del frontis indica que su construcción se realizó cuando ya existían desechos de material cerámico de estilo europeo, es decir, que su edificación fue posterior a 1533. Probablemente, en 1540, la iglesia ya había sido construida, tal como se deduce del reporte de fray Vicente Valverde en 1539 y de la designación en 1540 de Juan Funes como sacerdote de San Miguel. En 1543, el gobernador Vaca de Castro había definido la jurisdicción de los obispados de Lima, Cusco y Quito, en los cuales ya se habían fundado ciudades y construido iglesias. El período de 1543 a 1548 fue muy convulsionado para dedicarse a la construcción, pues, durante la rebelión de los encomenderos contra la Corona española, los antiguos centros incas de la región — como Piura La Vieja y Caxas- , así como la red vial inca, fueron utilizados por los rebeldes y las autoridades leales a la Corona. Entre 1548 y 1550, la iglesia de San Miguel ya estaba completamente operativa, tal como Cieza (1973 [1551]: 155) lo atestigua, y como aparece también en el testamento de Antón de Carrión en 1548 (Pavel Elías comunicación personal 2009).

El quinto detalle es una estructura arquitectónica de adobes registrada durante la temporada 2011, situada en el tercio anterior de la nave, con esquinas redondeadas y equidistante de los muros laterales de la iglesia (lo cual indica su articulación con la misma), donde se encontró la mayor concentración de cerámica colonial. Esta estructura ha desafiado nuestro supuesto que el piso de la nave era un espacio llano sin construcciones. Difícilmente, podría tratarse de la base del sotacoro, debajo del coro, pues está alejado de los pies de la iglesia.

La decisión de fundar una ciudad con su iglesia y organizar un obispado, luego de dos intentos frustrados, permite apreciar la necesidad hispana de establecer una iglesia que sirviese tanto para los españoles como para evangelizar a los indígenas por parte de los Mercedarios durante la década de 1540 (Elías 2008: 15). La primera etapa de la evangelización estuvo vinculada con las órdenes mendicantes y las encomiendas: los encomenderos a cambio de la mano de obra y tributo de sus encomendados debían evangelizarlos (Estenssoro 2003: 36-46; Elías 2008: 16). 


\section{El breve esplendor de San Miguel de Piura}

A pesar de que la década de 1550 fue más calmada, no se produjo la esperada consolidación de San Miguel de Piura como uno de los principales centros del naciente virreinato del Perú, sino todo lo contrario. Alrededor de 1550, Cieza de León estuvo en San Miguel y reclamó que la ciudad estaba «[...] en poca estimación por ser los repartimientos cortos y pobres [...]» (1973 [1551]: 157), y que merecía ser honrada y privilegiada por haber sido la primera avanzada española.

El breve esplendor de San Miguel y su iglesia matriz se debe al prestigio de sus habitantes: los encomenderos. En 1561, había 41 encomiendas en el corregimiento de San Miguel a cargo de 22 encomenderos y, en 1569, existían 23 encomenderos. Una de las principales actividades económicas de los encomenderos en San Miguel fue el comercio de aceite y vino provenientes de Panamá (Del Busto 1989), atestiguado por el registro de numerosos restos de jarras de oliva del estilo medio, producidas luego de 1560 (Goggin 1960: 11-17). El consumo de comida y bebida se realizó en una prestigiosa vajilla importada (probablemente, de Sevilla) de un solo tipo (estilo) de mayólica azul sobre blanco, al que hemos denominado «Piura La Vieja azul sobre blanco».

La ciudad acogió a visitantes ilustres y pudo presentar una planificación urbana completa. En 1557 y 1558, durante la visita del corregidor Diego de Pineda, se entrevistó a los curacas de Caxas en San Miguel. En su informe, se relatan los rituales en las edificaciones religiosas y la participación de los sacerdotes:

[...] Por tanto, que ruega y encarga al muy reuerendo señor Bachiler Juan López Guijarro, vicario de la iglesia desta ciudad, haga decir vna misa solene al Espíritu Santo, [...] E después de hauer dicho e acabado la misa en presencia de los susodichos, el dicho señor corregidor dijo que juraua y juró por Dios y por Santa María [...] Y [...] salieron en procesión con toda la gente que estaba, lleuando la cruz delante con la solenidad que de presente se pudo hacer. Y fueron en procesión a la Casa de Nuestra Seńora de la Merced desta ciudad. E allí se dijo misa de Nuestra Señora. E dicha voluieron en procesión a la dicha iglesia. Y allí el dicho señor vicario dijo ciertas oraciones invocando la gracia del Espíritu Santo al dicho efeto [...] (Pineda 1557 y 1558, citado en Espinoza 2006: 204-205).

No obstante, la iglesia matriz no solo fue el centro de las misas y procesiones religiosas, sino que también fue la última morada de algunos vecinos de San Miguel. Al menos, cuatro individuos fueron enterrados al interior de la iglesia siguiendo la tradición cristiana; uno de ellos fue registrado durante la temporada 2005 (Rodríguez 2006) y los otros tres durante la temporada 2008 (Astuhuamán 2012). Los cuatro comparten el estar dispuestos a lo largo del eje central de la edificación y, al menos, uno de ellos está orientado hacia el altar de la iglesia. Todos tuvieron nombre y apellido, y tal información nos ha llegado por los testamentos contenidos en los archivos. Uno de ellos fue Antón Carrión, quien pidió en 1548 ser enterrado en la iglesia; sin embargo, desconocemos su fecha de fallecimiento y si sus deudos cumplieron con su voluntad. Durante la temporada 2011, se han registrado las huellas de más entierros a lo largo del eje longitudinal de la iglesia: el piso de la iglesia fue roto para colocar los entierros, de modo que se privilegiaba la parte central de la edificación y la proximidad al altar.

El registro del individuo joven en la Cuadrícula 91 durante la temporada 2008, en el ingreso de la iglesia, ha permitido reconstruir el ritual de un entierro. Luego de morir, ser lavado, cambiado y velado, y de los rituales católicos en la iglesia, el piso blanco de la misma fue roto para cavar la fosa que contendría el cadáver y se extrajo el relleno sobre el cual se construyó el piso. El cuerpo fue colocado boca arriba y extendido en la matriz, probablemente, envuelto con un sudario, y empezó a ser cubierto con el mismo relleno extraído debajo del piso; un deudo arrojó un rosario mientras el cadáver era cubierto. Tras ser enterrado, el piso empezó a nivelarse nuevamente.

\section{El dramático colapso de San Miguel de Piura}

En 1565, se establecieron definitivamente los corregimientos en el virreinato del Perú. El corregimiento de Piura tuvo como centro a San Miguel y alrededor se ubicaban los distintos curacazgos que se habían entregado en encomiendas. Sin embargo, como ya lo indicaba Salinas de Loyola, en 
la década de 1560, San Miguel se estaba despoblando. Ello se expresa también en que el número de encomenderos disminuyó: en 1561, había 22 y casi la mitad en 1572, debido a las restricciones para obtener nuevas encomiendas instauradas por el virrey Toledo (Villanueva et al. 2002: 291; Elías 2008: 17, 22). En el aspecto religioso, la segunda etapa de evangelización está marcada principalmente por las políticas de Toledo, quien reguló la instalación de poblaciones locales en reducciones con una iglesia a cargo de un sacerdote doctrinario (clero secular), a partir de lo cual se fue dejando de lado gradualmente al clero regular (Elías 2008: 17-18). San Miguel de Piura fue perdiendo prestigio, pero no fue transformada en una reducción de indios. Su iglesia se mantuvo operativa hasta por lo menos 1572, pero con bachilleres como clérigos (ibid.: 22). Incluso, se produjo un traslado de la población en los primeros años de la década de 1560; no obstante, luego de dos años, volvieron a San Miguel.

Las excavaciones en la iglesia matriz muestran cómo se habría producido este gradual despoblamiento, que coincide con la fecha del abandono con los devastadores efectos de un mega Niño (ENSO), aquel de 1578 que está muy bien documentado etnohistóricamente (Hocquenghem 1998), pero que tiene escasos correlatos arqueológicos reportados.

El registro de la estratigrafía en las excavaciones muestra que, antes de las torrenciales lluvias, se produjo en la iglesia matriz un incendio de grandes proporciones (completamente silenciado en las fuentes escritas), pues las evidencias han sido registradas en diversas cuadrículas situadas tanto en la parte anterior, central y posterior del edificio. El incendio habría sido facilitado por los techos de madera y paja que cubrían la iglesia, como describió Salinas de Loyola: «[...] las cobijas [son] de paja, como llueve poco [...] En la dicha ciudad [de Sant Miguel] hay una iglesia de tanmano suficiente, trazada y edificada de los materiales dichos de las demás casas, que se edificó al principio que se pobló la dicha ciudad a costa de los vecinos y naturales. Hay también un monasterio de la Orden de Nuestra Señora de la Merced, edificado de la misma suerte y a costa de los dichos [...]» (Salinas de Loyola 1965 [1571], t. 2: 40-41).

La fecha tentativa del incendio podría situarse entre 1573 y 1578, pues, en 1573, se realizó un proceso judicial en contra de Joan de Saavedra, miembro del clero secular, quien estuvo temporalmente detenido en la sacristía de la iglesia (AGI 1574: Quito, 76, N. 11) . En dicho documento, no se menciona que la iglesia se haya quemado, puesto que, de ser así, difícilmente habría sido usada como el lugar de detención del temible Joan de Saavedra. La falta de previsión ante las intensas lluvias sugiere que, luego del incendio, la techumbre de la iglesia no fue bien reparada y que las lluvias hicieron colapsar el edificio. Abundantes tipos de clavos de metal y maderas quemadas, provenientes de la techumbre, fueron registrados durante las excavaciones. Esta sucesión de "castigos divinos» (fuego y diluvio) sobre la iglesia de San Miguel pudo ser el detonante para el abandono final de la segunda ciudad fundada por los españoles en el Perú. Otra explicación para el incendio es que este haya sido intencional, durante el proceso de desacralización de la iglesia que precedió al abandono de San Miguel; es decir, así como la iglesia fue consagrada a Dios mediante un ritual antes de 1540, también a través de un ritual de desacralización, que incluía un incendio controlado, se quemaron varios sectores de la iglesia, y otros permanecieron sin ser quemados como el altar escalonado y la sacristía.

Así, además del incendio, la dureza del clima, la persistencia de una enfermedad de la vista o «mal de ojos» (Cieza 1973 [1551]: 157), y las lluvias torrenciales que desintegraban las partes más descubiertas de las casas obligaron a los ya pocos habitantes de San Miguel a abandonarlas luego de 1578. A principios de la década de 1580, ya se había erigido provisionalmente un tercer emplazamiento de San Miguel, el de San Francisco de Buena Esperanza de Payta, hasta que se trasladó definitivamente al lugar que hoy ocupa en 1588 (Villanueva et al. 2002: 274).

Las excavaciones en la iglesia también muestran que esta se construyó en dos momentos. Se han registrado la sobreposición de dos pisos cerca al altar y fue sobre el último de ellos que se han registrado las huellas del incendio. También, se ha observado dos ingresos desde la nave hacia la sacristía, el segundo de los cuales clausuró al primero. La estructura arquitectónica de adobes con esquinas redondeadas, situada en el tercio anterior del interior de nave, podría tratarse de un tercer momento constructivo, pues rompe el patrón arquitectónico de la nave. Esto nos muestra la vigorosidad y vitalidad de los habitantes de San Miguel de Piura durante la segunda mitad del siglo XVI. 


\section{Conclusiones y perspectivas de investigación}

Las investigaciones en Piura La Vieja, como pocos sitios, han permitido examinar una larga secuencia de ocupación prehispánica y la temprana ocupación europea del sitio. Asimismo, han posibilitado descubrir, a partir de las evidencias materiales, los sucesivos gobiernos que desde este centro controlaron el Alto Piura. La evaluación del potencial arqueológico de los principales sectores de Piura La Vieja nos ha conducido a definir tres momentos de ocupación y sus respectivos correlatos materiales: preinca, inca y colonial transicional. Aunque nuestras investigaciones se han concentrado en los sectores excavados y en el Período Colonial Transicional, una mirada más allá de dichos sectores muestra las plataformas masivas típicas de las sociedades norteńas preinca (Sector MP-1), y también el patrón ortogonal y la plaza de traza triangular de tiempos inca.

La iglesia de San Miguel corresponde a las primeras ciudades fundadas por los españoles en América del Sur: San Miguel de Piura (1534), Cusco (1534), Quito (1534), Lima (1535), Trujillo (1535), Valparaíso (1536), Buenos Aires (1536), Popayán (1536), Asunción (1537), Santa Fe de Bogotá (1538), Guamanga (1539), La Plata (1539), y otras anteriores a 1570. Sin embargo, a diferencia de San Miguel, las iglesias de estas primeras ciudades fueron ampliadas y se realizaron varias modificaciones, por lo cual no había sido posible reconstruir su planta original, sus características arquitectónicas y artefactos asociados, y su proceso histórico vinculado a la temprana evangelización. La relevancia de la iglesia de San Miguel es que es la única de las siete primeras iglesias construidas en el Perú (Valverde 1939, citado en Vargas 1953: 120) cuyas evidencias han sido posibles registrar. El diseño arquitectónico de la iglesia es diferente al de las iglesias de reducciones de indios que caracterizan al período toledano. A partir de esta primera iglesia, es posible establecer una tipología de iglesias tempranas en el Perú y establecer una secuencia cronológica, posible de ser contrastada con otras propuestas para el área andina (v.g. Villacorta 1994; Wernke 2003, 2007; Mattos 2004).

La ocupación europea en Piura La Vieja podría ser definida como transicional (1532-1570), pues es anterior a las reformas toledanas que empezaron a consolidar el virreinato del Perú. Este carácter transicional se expresa en la iglesia excavada y en el material arqueológico recuperado. El registro ha sido facilitado por sus características pompeyanas, en particular, del Sector ES-1, cubierto por una capa de ceniza; luego, por una capa de sedimento producto de las intensas lluvias; y, finalmente, abandonado en 1578.

El análisis arquitectónico de los planos elaborados antes de la temporada 2008 permitió elaborar una hipótesis acerca de la ubicación la iglesia matriz de San Miguel de Piura. Por su parte, las investigaciones desarrolladas durante la temporada 2011 han permitido identificar la iglesia matriz de San Miguel de Piura y entender sus detalles atípicos. Quedan aún otros edificios, documentados por Salinas de Loyola en 1561 y en otras fuentes escritas examinadas por Pavel Elías, que no han sido identificados (v.g. el monasterio de los mercedarios). Así mismo, la localización de la iglesia en una hilera de edificios considerados sagrados ( v.g. Sector T y la plataforma escalonada al costado de la iglesia) sugiere que la iglesia fue situada en el sector religioso local, pero lejos del sector ritual inca.

En relación con la Casa de Nuestra Señora de la Merced, hacia donde las procesiones eran dirigidas desde la iglesia matriz (Pineda 1557 y 1558, citado en Espinoza 2006: 204-205), se debe anotar que este convento fue el otro centro religioso en San Miguel de Piura durante el Período Colonial Transicional, así que es posible que estuviese lejos de plaza central donde se localizaba la iglesia matriz. Probablemente, se habilitó en la kallanka, inca a un costado de la plaza triangular, debido a que dicho convento fue fundado en 1533 (Vargas 1953: 111; Aparicio 2001: 220), un año antes del establecimiento de San Miguel de Piura. La misma situación ocurrió en Lima entre 1534 y 1535. Así, en 1540, los mercedarios tenían cuatro casas en Perú: San Miguel de Piura, Lima, Cuzco y Guamanga (Vargas 1953: 111, 217). Futuras excavaciones en la kallanka proporcionarán evidencias para validar esta hipótesis. La Orden de la Merced fue religiosa y guerrera; sus integrantes acompañaron a los primeros conquistadores y ellos utilizaron los edificios incas disponibles para 
transformarlos en conventos e iglesias, situación que fue aceptada y reglamentada por el Primer Concilio de 1551, pues la Constitución Segunda dispuso que «[...] las iglesias se levantaran en el pueblo principal donde tenía su ordinario asiento el cacique principal [...]» (Vargas 1953: 128).

Las excavaciones en la Estructura Singular 1 han permitido exponer muros, pisos, retablos, altares que conforman la arquitectura religiosa de la iglesia, e incluso ubicar algunos entierros debajo del piso de la nave. Estos diversos tipos de evidencias y su precario estado de conservación requieren de diferentes medidas de protección y conservación con miras a la posterior exposición al público de esta iglesia. En lo que respecta a los últimos años (2005-2012), las investigaciones realizadas han permitido conocer la fundación, esplendor y colapso de la edificación más importante de San Miguel de Piura, y, en los próximos ańos, con su puesta en valor y apertura al turismo, se conseguirá que Piura La Vieja logre el desarrollo que anhela.

\section{Agradecimientos}

El Proyecto de Investigación Arqueológica «San Miguel de Piura», desarrollado durantelas temporadas 2005, 2008 y 2011, fue parte del Proyecto de Investigación Histórica, Arqueológica, Arquitectónica y Urbana «San Miguel de Piura, Primera Fundación Española en el Perú (1534)». La ejecución de este proyecto fue posible gracias al financiamiento del Programa de Cooperación Científica con Iberoamérica de la Universidad Politécnica de Madrid, la colaboración de la Universidad de Piura y de la Fundación Diálogos (España), en el marco de un Proyecto General de Cooperación Científica entre ambas universidades, así como el apoyo económico de la Municipalidad Distrital de La Matanza. Durante las temporadas 2005, 2008 y 2011, participaron alumnos de la Escuela de Arqueología de Universidad Nacional Mayor de San Marcos. A todos ellos quiero expresarle nuestro agradecimiento, en especial, al doctor Fernando Vela Cossio, director científico del proyecto.

\section{FUENTES MANUSCRITAS}

Archivo General de Indias (AGI). Sevilla

1574 Quito, 76, N. 11. Cartas y expedientes del obispo de Quito. Testimonio sobre el pleito entre el padre Hernán Saco de Quiroga, vicario de la ciudad de San Miguel de Piura contra el padre Juan de Saavedra. Cuenca. 72 fols.

\section{REFERENCIAS}

Aparicio, S.

2001 La orden de la Merced en el Perú; Estudios Históricos, tomo 1, Provincia Mercedaria del Perú, Cuzco.

Astuhuamán, C.

2008 The organization of the Inca provinces within the highlands of Piura, northern Peru, tesis de doctorado, Institute of Archaeology, University College London, Londres.

2009 Informe final del Proyecto Investigación Arqueológica San Miguel de Piura: Primera fundación española en el Perú (1534), temporada 2008, Instituto Nacional de Cultura, Lima.

2012 Informe final del Proyecto Investigación Arqueológica San Miguel de Piura: Primera fundación española en el Perú (1534), temporada 2011, Instituto Nacional de Cultura, Lima.

Astuhuamán, C., N. Guzmán, y J. León

2003 Informe consolidado final Macro Región Norte: Proyecto levantamiento de información del sistema Vial Inca - Qhapaq Nan, Instituto Nacional de Cultura, Lima.

Cieza de León, P. de

1973 La crónica del Perú, Peisa, Lima.

[1551]

1997b La crónica del Perú. Tercera parte, Pontificia Universidad Católica del Perú, Lima.

[1554] 
Cornejo, M.

2000 La nación Ichma y la provincia Inca de Pachacamac, Arqueológicas 24, 149 -173.

\section{D’Altroy, T. N.}

1992 Provincial power in the Inka Empire, Smithsonian Institution Press, Washington, D.C./Londres.

Deagan, $\mathrm{K}$.

1996 Colonial transformation: Euro-American cultural genesis in the early Spanish-American colonies, Journal of Anthropological Research 52 (2), 135-160. https://doi.org/10.1086/jar.52.2.3630198

\section{Deagan, K. y J. Cruxent}

2002 Archaeology at La Isabela: America's first European town, Yale University Press, New Haven /Londres. https://doi.org/10.12987/yale/9780300090413.001.0001

Del Busto, J. A.

1989 Los trece de la fama, Studium, Lima.

Duviols, P.

1977 La destrucción de las religiones andinas (durante la Conquista y la Colonia), Universidad Nacional Autónoma de México, México DF.

Elías, P.

2008 Piura y su jurisdicción religiosa: siglos XVI y XVII, Evangelización y vida eclesial en Piura: Siglos XVI y XVII, Cuadernos de Humanidades 14, 13-38, Universidad de Piura, Piura.

Eliade, M.

1998 Lo sagrado y lo profano, Editorial Paidós, Barcelona.

Espinoza, W.

2006 La etnia guayacundo en Ayabaca, Huancabamba y Caxas (siglos XV-XVI), Fondo Editorial del Pedagógico San Marcos, Lima.

Estenssoro, J. C.

2003 Del paganismo a la santidad: La incorporación de los indios del Perú al catolicismo 1532-1750, Pontificia Universidad Católica del Perú/Instituto Francés de Estudios Andinos, Lima. https://doi.org/10.4000/ books.ifea. 4412

\section{Goggin, J.}

1960 The Spanish olive jar: An introductory study, Yale University Publications in Anthropology 62, Yale University Press, New Haven.

Hocquenghem, A.-M.

1998 Para vencer la muerte. Piura y Tumbes. Raices en el bosque seco y en la selva alta, horizontes en el Pacifico y en la Amazonía, CNRS/IFEA, Lima.

Huertas, L.

1996 Patrones de asentamiento poblacional en Piura (1532-1850), Boletín del Instituto Francés de Estudios Andinos 25 (1), 91-124.

Hyslop, J.

1990 Inka settlement planning, University of Texas Press, Austin.

Matos, R.

1994 Pumpu: Centro administrativo Inka de la puna de Junin, Horizonte, Lima.

Mattos, L.

2004 Urbanismo andino e hispanoamericano: Ideas y realizaciones (1530-1830), Fondo Editorial FAUA, Lima.

Montenegro, J.

2005 Informe final. Proyecto Arqueológico Chulucanas - Morropón, valle del Alto Piura, departamento de Piura, documento presentado a la Comisión Nacional Técnica de Arqueología, Instituto Nacional de Cultura, Lima.

2010 Interpreting cultural and sociopolitical landscapes in the upper Piura Valley, far North Coast of Peru (1100 B.C.-A.D. 1532), tesis del doctorado, Departamento de Antropología, Southern Illinois University Carbondale, Carbondale.

Morris, C.

1998 Inka strategies of incorporation and governance, en: G. Feinman y J. Marcus (eds.), Archaic states, 293-309, School of American Research Press, Santa Fe. 
Oliva, O.

1998 Historia del reino y provincias del Perú, Pontificia Universidad Católica del Perú, Lima.

[1630]

Rappaport, R.

2001 Ritual y religión en la formación de la humanidad, Cambridge University Press, Cambridge.

Renfrew, C.

1975 Trade as action at a distance: Questions of integration and communication, en: J. A. Sabloff y C.C. Lamberg-Karlovski (eds.), Ancient civilization and trade, 3-59, University of New Mexico, Albuquerque.

Renfrew, C. y P. Bahn

1998 Arqueología: Teorías, métodos y prácticas, Editorial Akal, Madrid.

Renfrew, C. y E.V. Level

1979 Exploring dominance: Predicting polities from centers, en: C. Renfrew y K. Cooke (eds.), Transformations: Mathematical approaches to culture change, 145-167, Academic Press, New York/Londres.

Rodríguez, A.

2006 Informe final del Proyecto de Proyecto de Investigación Arqueológica San Miguel de Piura; Primera fundación española en el Perú (1534), manuscrito en posesión del autor.

Salinas de Loyola, J.

1965 Relación de la ciudad de Saint Miguel de Piura, en: M. Jiménez de la Espada (ed.), Relaciones geográficas

[1571] de Indias, tomo 2, 35-45, Atlas, Madrid.

Schülke, A.

1999 On christianization and grave-finds, European Journal of Archaeology 2 (1), 77-106. https://doi. org/10.1179/eja.1999.2.1.77

Van Buren, M., P. Bürgi y P. Rice

1993 Torata Alta: A Late highland settlement in the Osmore Drainage, en: M. Aldenderfer (ed.), Domestic architecture, ethnicity and complementarity in the South-Central Andes, 136-146, University of Iowa Press, Iowa City.

Vargas Ugarte, R.

1953 Historia de la Iglesia en el Perú, 1511-1568, tomo I, Santa María, Lima.

Vela Cossío, F.

2000 Investigación histórica y arqueológica en San Miguel de Piura. Primera fundación española en el Perú, Revista de Arqueología 21 (233), 55-58.

Vela Cossío, F. y L. Villanueva Domínguez

2002 Puesta en valor de la ciudad de San Miguel de Piura, primera fundación española en el Perú, Boletín del Instituto Andaluz de Patrimonio Histórico 39, 8-9.

Villacorta, L. F.

1994 Iglesias rurales en el valle de Lima durante la Colonia (1534-1821), Boletín de la Sociedad Geográfica de Lima 107, 47-66.

Villanueva, L. de

2001 La construcción de San Miguel de Piura, primera fundación española en el Perú. Conferencias y artículos, CIE Dossat/Fundación Diálogos, Madrid.

Villanueva, L. de, F. Vela Cossío, A. Navarro y D. Rivera

2002 La ciudad de San Miguel de Piura, primera fundación española en el Perú, Revista Española de Antropología Americana 32, 267-294.

Wernke, Steven A.

2003 An archaeo-history of Andean community and landscape: The late prehispanic and early colonial Colca Valley, Peru, tesis de doctorado, Departamento de Antropología, University Of Wisconsin, Madison

2007 Analogy or erasure? Dialectics of religious transformation in the early Doctrinas of the Colca Valley, Peru, International Journal of Historical Archaeology 11 (2), 152-182. https://doi.org/10.1007/s10761007-0027-5

Williams, V. I.

2002- Provincias y capitales: Una visita a Tolombon, Salta, Argentina, Xama 15-18, 177-198.

2005 